\title{
Olympiades de Physique France XXVIII concours national
}

\begin{abstract}
Cette année fêtait la XXVIII édition de la finale du concours des Olympiades de Physique France. La manifestation s'est déroulée malgré les contraintes liées aux mesures sanitaires. L'Université de Bordeaux a - virtuellement - accueilli les 29 et 30 janvier 2021 les équipes sélectionnées, à l'invitation de la section locale Aquitaine de la Société Française de Physique et de la section académique de l'Union des Professeurs de Physique et de Chimie.
\end{abstract}

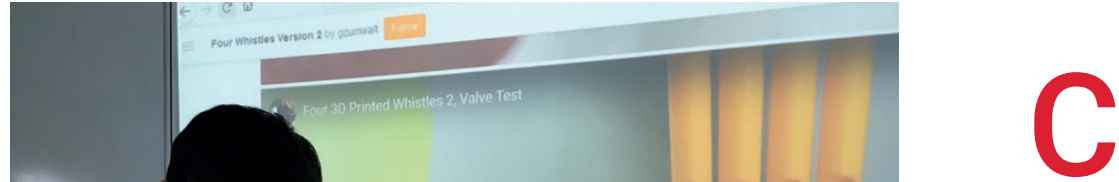

C ette finale nationale a rassemblé vingt-trois équipes sélectionnées en France et de sections francophones de lycées à l'étranger. Elle a été parrainée par Jean-François Clervoy, ingénieur et spationaute à l'Agence Spatiale Européenne, président d'honneur de la société Novespace. Toutes les épreuves se sont tenues à distance afin de respecter les contraintes des protocoles sanitaires. Parmi les projets retenus pour la finale nationale, voici deux exemples de prestations de grande qualité couronnées d'un premier prix pour le remarquable travail expérimental effectué.

\section{Organettissimo}

Ce projet a été présenté par les élèves de première, Klara Demarle, Narhan Senenoff, El-Hamid Ghout et Nicolas Berlemont, encadrés par Sophie Gronlier du lycée Van der Meersch à Roubaix.

Construire un orgue miniaturisé, voilà le défi que ces jeunes et téméraires élèves se sont donné. Pour cela, il faut concevoir des modèles de tuyaux sonores, en étudier les propriétés, concevoir des embouts de qualité, trouver une imprimante 3D pour créer un prototype et tester le tout (sur un air de Harry Potter).

Les élèves ont commencé par étudier la résonance de tuyaux cylindriques en fonction de leur longueur pour la gamme tempérée.

Mais la réalité s'est révélée plus compliquée. Il faut aussi tenir compte du système excitateur et créer une lame d'air qui viendra exciter le tuyau sonore par le biais d'un biseau. Le son est produit par le jet d'air, expulsé par la « lumière " de l'instrument - à savoir l'orifice de sortie de la lame d'air -, qui va osciller autour de la lèvre du biseau; le tuyau cylindrique sert de résonateur à travers la colonne d'air qu'il contient. 


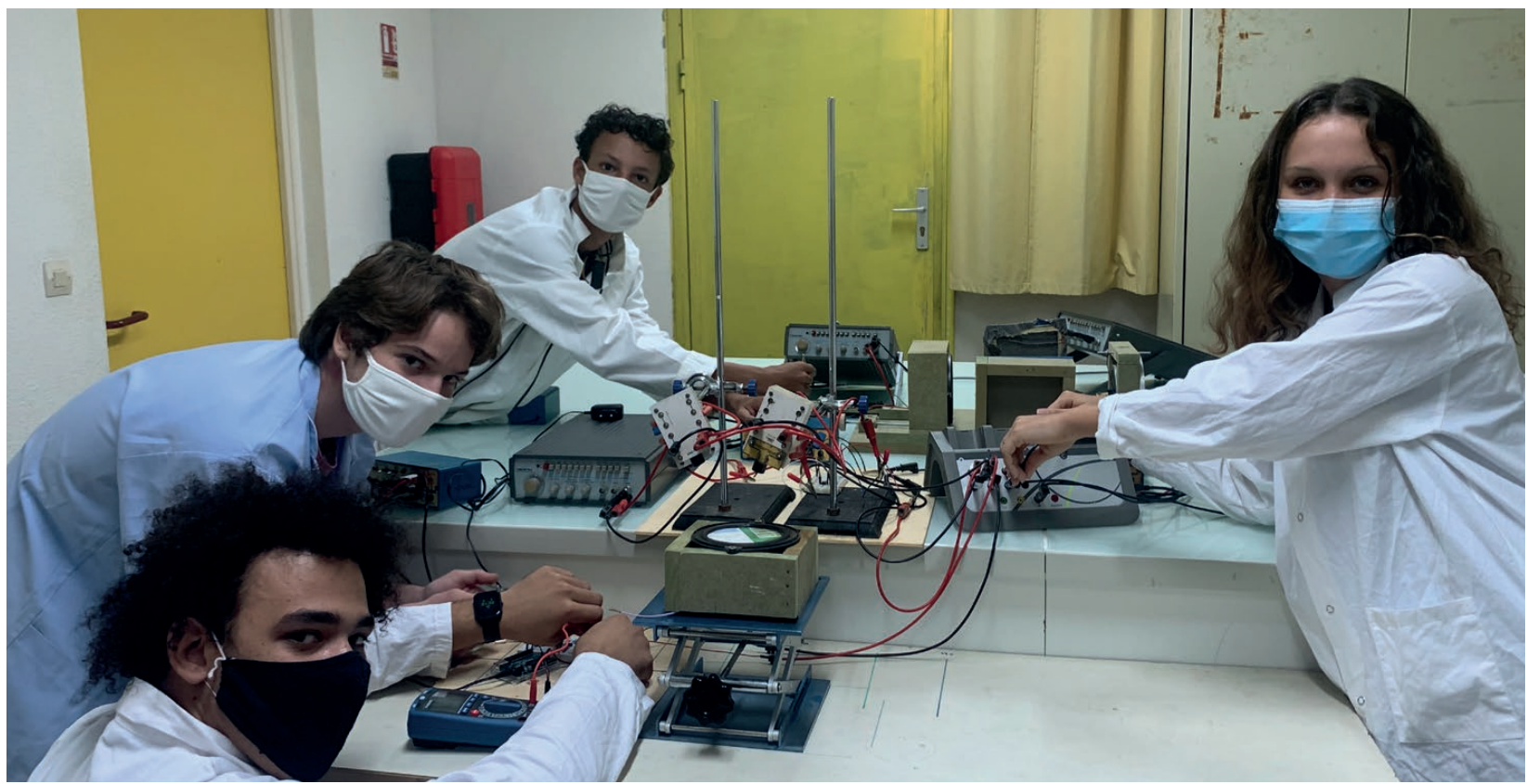

2. L'équipe du lycée Yves Leborgne, en plein travail.

Les phénomènes acoustiques les plus importants se situent dans l'espace situé entre la « lumière " et la pointe du biseau. C'est là que se passent les interactions entre le filet d'air excitateur et la colonne d'air du tuyau. La forme de la lumière et la distance entre la lumière et la pointe du biseau sont les principaux paramètres.

Les élèves font donc évoluer la forme de la lumière d'un demi-cercle à un croissant de lune et déterminent la forme optimale du croissant leur permettant d'obtenir le meilleur niveau sonore. Puis, ils cherchent quel décalage choisir entre la lumière et l'axe du canal afin de trouver les conditions optimales à l'émission d'une note. Enfin, ils examinent l'influence de la longueur de la fenêtre, à une fréquence donnée par la longueur du tuyau.

Il ne restera " plus qu'à " réaliser ce petit orgue (fig. 1), après avoir fabriqué les embouts adaptés à chaque tuyau leur permettant de jouer les quatre notes de leur mélodie.

\section{Mesure de battements cardiaques à distance}

Ce projet a été présenté par les élèves : Adèle Piquet, Antoine Pinard, Aurélien Trouillefou et Hector Panabières, encadrés par leur professeur, Jean-Pierre Pichou, du lycée Yves Leborgne à Sainte-Anne, Guadeloupe.

Le but que se sont donnés ces élèves est de détecter les battements du cœur à partir des infimes mouvements corporels qu'ils engendrent. Pour cela, ils ont d'abord réalisé un simulateur des mouvements du thorax engendrés par le cœur, en utilisant la membrane d'un haut-parleur excitée par un signal périodique électrique « carré » de $1 \mathrm{~Hz}$.

Dans une première étude, à partir de l'accéléromètre de leur téléphone, les signaux des mouvements enregistrés sont traités dans un tableur afin d'obtenir, par calcul, l'allongement normal de la membrane du haut-parleur en fonction du temps, lié aux battements cardiaques. Mais le résultat n'est pas probant.

Une autre méthode de détection des mouvements de la membrane utilise un interféromètre de Michelson pour des ondes ultra-sonores (fig. 2). L'onde quasi plane nécessaire aux interférences est obtenue en plaçant la source d'ultrasons au foyer d'un miroir sphérique, puis elle est séparée en deux ondes planes grâce à un miroir semi-réfléchissant, dont les élèves choisissent le matériau. L'une des deux ondes sera réfléchie par un miroir placé sur la membrane, l'autre par un miroir fixe. Les deux ondes planes recombinées sont focalisées sur le détecteur, et le déphasage entre le faisceau de référence et le faisceau réfléchi par la paroi mobile modifie l'intensité du faisceau émergent et indique directement les déplacements de cette paroi, rendant compte des battements cardiaques. Avec une longueur d'onde ultra-sonore de $8,8 \mathrm{~mm}$, il est aisé de détecter l'amplitude du signal final avec une bonne sensibilité : nos élèves peuvent mesurer ici un déplacement de 0,15 mm.

Mais cette équipe, experte en électronique, ne s'arrête pas là. Ils essaient une troisième expérience, consistant à mettre en évidence le déphasage entre le signal de l'émetteur et celui réfléchi par la paroi mobile. En mesurant ce déphasage, on obtient les déplacements de la paroi.

Pour ces élèves, il leur restera à étudier de plus près comment intégrer la reconnaissance à distance par les caractéristiques des battements cardiaques qui sont propres à chacun. I

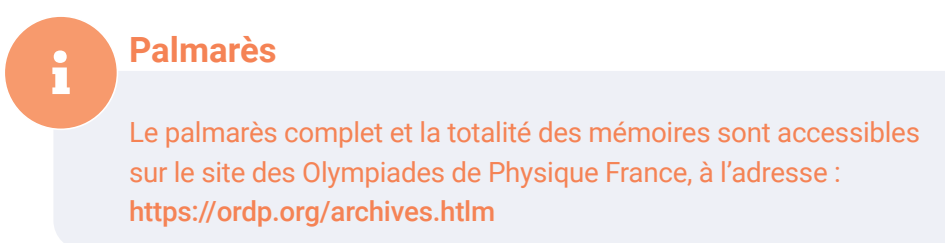

